\section{Buchrezension zu: Strasburger}

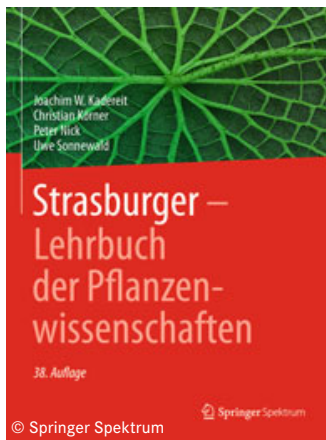

Strasburger -

Lehrbuch der

Pflanzenwissenschaften

J. W. Kadereit, C. Körner, P.

Nick und U. Sonnewald (Hrsg.)

1555 S., 407 sw. Abb., 641 farb. Abb., Springer Spektrum, 38. Auflage, 2021. HC, 84,99€.

ISBN: 9783662619421

Auch als E-Book erhältlich

DOI: 10.1007/s12268-021-1681-6 (C) Der Autor 2021

Die 38. Auflage des Standardwerks der Pflanzenwissenschaften ist eine aktualisierte Fassung der 37. Auflage (2014) oder der damals gründlich überarbeiteten 36. Fassung aus dem Jahr 2008. Die Autoren verfolgen konsequent die Tradition des „Strasburgers", die Pflanzenwissenschaften in der ganzen Breite zu präsentieren. Beibehalten wurde daher weitgehend die bisherige Gliederung, sodass die Grundlagen der grünen Biologie in bekannt umfassender Weise dargestellt werden. Neu dagegen ist, dass die Autoren die Lesenden deutlich stärker an den Stand der aktuellen Forschung heranführen.

Diese stärkere Forschungsorientierung ist eine Herkulesaufgabe, wenn man sich vergegenwärtigt, dass im Jahr 2000 ca. 7.000 Publikationen im Bereich der Pflanzenwissenschaften erschienen sind, diese Zahl im Jahr 2008 auf 67.100 stieg und im Jahr 2020 die Zahl von 126.000 erreichte. Diese Zahlen belegen, warum die Autoren erstmals im Vorwort engagiert auf die wachsende Bedeutung der modernen
Pflanzenwissenschaften für die Zukunftssicherung der Menschheit hinweisen. Ohne Fortschritte in den Pflanzenwissenschaften wird die Sicherstellung der Ernährung von acht bis zehn Milliarden Menschen nicht gelingen und ohne ein tieferes Verstehen der Stoffflüsse, die in der Biosphäre durch die Pflanzenwelt bestimmt werden, fehlen den Klimawissenschaftler:innen grundlegende $\mathrm{Da}$ ten. Daher wurden in der neuen Auflage die besonders schnell wachsenden Forschungsfelder, wie die Genomeditierung be Pflanzen, die Epigenetik und Gentechnik, neu und ausführlicher gefasst. Auch die Antwort der Pflanzen auf Klima- und Bodenfaktoren werden umfassender und expliziter und auf dem aktuellen Forschungsstand dargestellt.

Trotz dieser Erweiterung entspricht der Seitenumfang mit ca. 1.150 Seiten in etwa dem de 36. Auflage. Daher musste verständlicherweise auch gekürzt werden. So wurde z. B. das einleitende Kapitel zur Geschichte der Botanik und zur naturwissenschaftlichen Erkenntnistheorie durch eine Tabelle ersetzt, wann welche grundsätzliche Erkenntnis gewonnen oder Methode entwickelt wurde. Das ist zwar eine sehr hilfreiche Verdichtung von Information, aber es erklärt nicht den theoretischen Hintergrund. Ich empfinde das als Verlust, weil heute gerade Lehramtsstudierende gut auf wissenschaftstheoretische Diskussionen vorbereite sein sollten, da sie in der Schule auf eine Öffentlichkeit reagieren müssen, in der pflanzliche Gentechnik mit falschen Argumenten kritisiert wird, oder im Zuge des Klimawandels weniger naturwissenschaftliche als esoterische Sichtweisen als Argumente benutzt werden. Nur wenn der naturwissenschaftliche Hintergrund der Pflanzenwissenschaften klar ist, kann mach auch verstehen, warum in den Kapiteln Stoffwechsel, Entwicklung, Umweltinter- aktionen die zugrunde liegenden Mechanismen erläutert werden und nicht nur Effekte phänotypisch beschrieben werden. Dies spiegelt die Aktivität der heutigen Forschungslandschaft wider, in der die Aufklärung der Mechanismen im Vordergrund steht, um wissensbasiert in die Umwelt steuernd eingreifen zu können.

Die Autoren haben die gute Strasburger Tradition beibehalten, die Lesbarkeit und Verständlichkeit durch eine optimale Wahl von Bildern, Schemata oder Lernboxen zu unterstützen, wobei sehr oft Abbildungen aus Originalarbeiten übernommen und didaktisch verbessert wurden. Der die Abbildungen erläuternde Text ist durchgehend bestens verständ lich und macht das Werk zu einem Lehrbuch, das es auch dem Autodidakten erlaubt, komplexe Inhalte leicht zu verstehen. So entstand trotz der Verdichtung der Information zumeist kein Verlust an Verständlichkeit.

Am Ende jedes Kapitels werden die wichtigsten Arbeiten zitiert. Leider fehlen häufig im Quellenverzeichnis die Arbeiten, aus denen die modifizierten Abbildungen übernommen wurden. Vorbildlich dagegen ist das sehr umfangreiche Stichwortverzeichnis, das es dem Lesenden sehr leich macht, gesuchte Informationen zu finden.

Insgesamt ist das Buch allen Studierenden in verschiedenen Modulen der grünen Biologie zu empfehlen, angefangen von der Allgemeinen Botanik über Pflanzenphysiologie, Zellbiologie oder Ökologie bis hin zur Systematik. Für die Lehrenden in der gymnasialen Oberstufe oder im biologischen Grundstudium ist der neue Strasburger eine sichere und umfassende Informationsquelle. Der Preis ist angemessen und gerade das E-Book oder die digitale pdfVersion erlauben den leichten Zugriff auf das Lehrbuch.
Christian Wilhelm, Universität Leipzig, cwilhelm@rz.uni-leipzig.de

Diese Rezension erscheint Open Access.

$$
\begin{aligned}
& \text { Funding note: Open Access funding } \\
& \text { enabled and organized by Projekt DEAL. } \\
& \text { Open Access: Dieser Artikel wird unter } \\
& \text { der Creative Commons Namensnen- } \\
& \text { nung } 4.0 \text { International Lizenz veröffent- } \\
& \text { licht, welche die Nutzung, Vervielfälti- } \\
& \text { gung, Bearbeitung, Verbreitung und } \\
& \text { Wiedergabe in jeglichem Medium und } \\
& \text { Format erlaubt, sofern Sie den/die ur- } \\
& \text { sprünglichen Autor(en) und die Quelle } \\
& \text { ordnungsgemäß nennen, einen Link zur } \\
& \text { Creative Commons Lizenz beifügen und } \\
& \text { angeben, ob Änderungen vorgenommen } \\
& \text { wurden. Die in diesem Artikel enthalte- } \\
& \text { nen Bilder und sonstiges Drittmaterial } \\
& \text { unterliegen ebenfalls der genannten } \\
& \text { Creative Commons Lizenz, sofern sich } \\
& \text { aus der Abbildungslegende nichts ande- } \\
& \text { res ergibt. Sofern das betreffende Mate- } \\
& \text { rial nicht unter der genannten Creative } \\
& \text { Commons Lizenz steht und die betref- } \\
& \text { fende Handlung nicht nach gesetzlichen } \\
& \text { Vorschriften erlaubt ist, ist für die oben } \\
& \text { aufgeführten Weiterverwendungen des } \\
& \text { Materials die Einwilligung des jeweiligen } \\
& \text { Rechteinhabers einzuholen. Weitere De- } \\
& \text { tails zur Lizenz entnehmen Sie bitte der } \\
& \text { Lizenzinformation auf } \\
& \text { http://creativecommons.org/licenses/ } \\
& \text { by/4.0/deed.de. }
\end{aligned}
$$

